\title{
Surface Graft Polymerization of Acrylamide onto Plasma Activated Nylon Microfiber Artificial Leather for Improving Dyeing Properties
}

\author{
Shu-Chuan Liao, Ko-Shao Chen, Wei-Yu Chen, Chin-Yen Chou, and Kan-Chi Wai
}

\begin{abstract}
To improve surface wettability and dye-ability of Nylon microfiber artificial leather, we apply oxygen plasma treatment and subsequently graft-polymerize acrylamide (AAm) on the surface. The surface properties of AAm-grafted Nylon microfiber artificial leather are characterized by FT-IR, SEM, ESCA and dyeing density (C.I.reactive Blue 4). The dyeing rate of AAm-grafted Nylon microfiber artificial leather, up-regulated with higher acrylamide grafting concentration, increases to $67.1 \mathrm{mg} / \mathrm{cm}^{2}$, and remains $66.4 \mathrm{mg} / \mathrm{cm}^{2}$ after rinsed by water, showed better stability than that of control and oxygen plasma modified Nylon microfiber artificial leather. The water-absorptivity of s-graft acrylamide is five times than that of control. From the results, the efficiency and density of dyeing are both improved, indicating that plasma graft polymerization process could contribute to promote the absorption of dye on the artificial leather.
\end{abstract}

Index Terms -Plasma, surface graft, artificial leather, dye.

\section{INTRODUCTION}

Recent, consciousness of wildlife protection rises around the world. The usage of genuine leather is getting replaced by the developing of artificial leather. Micro-fiber that the textile industry circles invented is similar to genuine leather no matter on its outward appearance, characters or touching feels However, it has a problem of color deepen and fasten in the dyeing processes [1]-[3]. Surface modification plays a very important role in many applications involving various fields. Many methods have been considered and developed to alter the interactions of materials with their environments. In addition to keeping warm, microfiber has the advantage of softness and shining surface. But microfiber must use large amount of dye, because the textile fiber does not take dye well and fades easily [4], [5]. Now that the thinness of the textile fiber becomes more and more thin, i.e. the textile fiber has greatly increased surface area ratio per fiber unit. Accordingly, compared with common textile fiber, the adhesion of dye will become worse for thin textile fiber. Recently, surface modification treatments are often used to solve these

Manuscript received January 21, 2013; revised March 22, 2013

Shu Chuan Liao is with the Center Thin Film Technologic and Applications, Ming Chi University of Technology, New Taipei City, Taiwan (e-mail:scliao@mail.mcut.edu.tw).

Ko-Shao Chen and Chin-Yen Chou are with the Department of Materials Engineering, Tatung University, 40, Chungshan N. Rd., 3rd Sec.,Taipei, Taiwan (e-mail: kschen@ttu.edu.tw).

Wei-Yu Chen is with the Institute of Biomedical Engineering, National Taiwan University, Taipei, Taiwan.

Kan Chi Wai is with the Institute of Textiles and Clothing, The Hong Kong Polytechnic University. problems by plasma [6]. Plasma deposited film has many advantages, including good adhesion to most substrates, excellent uniformity, thickness control easily, easy to prepared, low porosity, no pin-hole formation, to provide some free radicals and peroxide groups. Free radicals formed on the surface of polymers and other solid materials exposed to plasma can be utilized to initiate graft polymerization in a manner that is similar to pre-irradiated grafting. Although the surface properties (wettability, electricity or photo-reactivity) of polymers can be improved after plasma treatment due to the formation of the induced polar groups and free radicals, this activated surface is not stable because the free radicals on surface would readily react with oxygen atoms in the air. No matter whether the textiles are hydrophilic or hydrophobic before modification, surface treatment by plasma will improve adhesion of dye and repellency properties [4]-[9]. Compared with other treatment processes, surface treatment by plasma will not cause damage to products, and will not restrict by surface chemistry and topography of modified material. In this study, modified the nylon microfiber artificial leather by cold plasma deposition system will form peroxides on surface, and then graft polymerization using various concentration of acrylamide solution by UV light. After graft polymerization, the specimens were cleaned with deionized water for one day and dried at room temperature. At last the specimens were spreader and immersed in the dye (concentration of dye is $3 \%$ owf). In order to evaluate the adhesive percentage of dye (dyeing density), the weight of specimens maybe increase in proportion to the amount dye adhered to specimens. Besides, the functional group of neutral-dyeing (C.I.reactive Blue 4) may analyze by Electron Spectroscopy for Chemical Analysis (ESCA) if neutral-dyeing (C.I.reactive Blue 4) adhered to specimens. After dyeing, the morphology and micro-structure of specimens were characterized by Scanning Electron Microscope (SEM).

\section{EXPERIMENAL}

\section{A. Substrate and Monomer}

Nylon microfiber artificial leather: textile was produced from Sanfang Ltd (Taiwan), and then cut the substrate up to the fitting size of $3 \mathrm{~cm} \times 3 \mathrm{~cm}$.

Acryl amide (C2H3CONH2), 95\%, Mw=71.08 g/mol, was obtained from Wako Pure Chemical Co. Japan.

Oxygen $\left(\mathrm{O}_{2}\right)$, purity $>99 \%$, Sanfu Chemical Co. (Taiwan)

The textile dye reactive blue 4 obtained from Sanfang Ltd 
Taiwan.

\section{B. Oxygen Plasma Treatment}

Substrate was individually put into the cylindrical reactor with a parallel electrode. The plasma reactor, Model manufactured by Taiwan, was pumped to a base pressure of 30 mTorr prior to plasma treatment. Ratio frequency of 13.6 $\mathrm{MHz}$ was used in this study and an impedance matching unit was required. After the pressure was adjusted to a stable desired working pressure (40 mTorr) by continuous flow of the oxygen gas, the plasma treatment condition was under 100 $\mathrm{W}$ for $1 \mathrm{~min}$. Substrate temperature was kept at room temperature during the process.

\section{UV-induced Surface Graft Polymerization}

After plasma treatment the surface of specimens contained several activated species such as electrons, ions, free radicals and some neutral species. Those active species may react with the oxygen and moisture in the atmosphere and form peroxides. The peroxides could further initiate surface graft polymerization induced by hydrophilic monomers under UV light exposure. Then, the graft polymerization was performed under UV light exposure (power: $1000 \mathrm{~W}$; wavelength: 365 $\mathrm{nm}$, Henchman Co. Inc., Taiwan). After graft polymerization reaction, the grafted specimens were washed with distilled water overnight to remove the homopolymer and residues, and finally dried at room temperature. The different concentration of acryl amide solution was 1 and $10 \mathrm{wt} \%$ and the grafting time was fixed at 40 minutes.

\section{Dyeing Process}

Reactive blue 4-dye concentration of $3 \%$ owf was used for microfiber dyeing. Solutions were prepared by dissolving requisite quantity of the dye in double distilled water. Treatment condition: $40^{\circ} \mathrm{C}$, 1 hour

\section{E. Characterization of the Nylon Microfiber Artificial Leather}

The infrared spectroscopy (Micro FTIR, Jasco type MFT-2000) was taken to characterize the chemical structure of the surface of modified films by reflection way. The spectra range of 400 to $4000 \mathrm{~cm}^{-1}$ at a resolution of $2.0 \mathrm{~cm}^{-1}$. Surface morphologies of the sensor materials were examined by scanning electron microscope (JEOL JSM-6300). First of all, the samples were placed on an aluminum holder and coated with a thin layer of gold (coating $5 \mathrm{~min}$ ) by sputtering to improve the electric conductivity. By ESCA (XPS) analysis, the chemical compositions of deposited films on substrate surface can be obtained. Spectra were recorded with a Perkin-Elmer PHI 590AM spectrometer by using $\mathrm{MgK} \alpha$ exciting radiation. Typical operating conditions were $\mathrm{x}$-ray gun, $15 \mathrm{kV}, 250 \mathrm{~W}$, and the $10^{-10}$ Torr pressure of the test chamber.

\section{RESULTS AND DISCUSSION}

\section{A. Wettability of Modified Surface}

The water-absorptivity of nylon microfiber artificial leather with different treatments was measured in this experiment. The results showed that grafting $10 \mathrm{wt} \%$ of acryl amide solution was more absorbent than untreated. After absorbing for 15 minutes, the water-absorptivity achieved five times than untreated substrates. According to literature, the reaction of low pressure $\mathrm{O}_{2}$ plasma on an organic surface involves two processes: surface etching and surface oxidation. Furthermore, in the low-pressure $\mathrm{O}_{2}$ discharge, there were some species for surface treatment: atomic oxygen, charged particles and UV radiation [2], [9]. We measured the water-absorptivity after different treatment and dyeing process may promote hygroscopic.

\section{B. References FTIR Characterization of Nylon Microfiber Artificial Leather Structure}

The FTIR spectrum of nylon microfiber artificial leather obtained via AAm grafting, and dye of reactive Blue 4 was shown in Fig.1 (a) unmodified, (b) after $\mathrm{O}_{2}$ plasma modification, (c) after $\mathrm{O}_{2}$ plasma treatment then grafting acryl amide $1 \mathrm{wt} \%$, and (d) $10 \mathrm{wt} \%$. As can be seen, oxygen-containing functional peaks appeared because the acryl amide bonding was broken by photo and then combined with the peroxides formed on the specimens by plasma treatment. It could be observed that there were several adsorption peaks of nylon microfiber artificial leather film appearing or enhanced such as $\mathrm{CH}_{2}\left(700 \sim 900 \mathrm{~cm}^{-1}\right), \mathrm{C}=\mathrm{C}$ $\left(1600 \mathrm{~cm}^{-1}\right), \mathrm{C}-\mathrm{O}\left(1125 \mathrm{~cm}^{-1}\right), \mathrm{C}-\mathrm{H}\left(1424 \mathrm{~cm}^{-1}\right)$, and $\mathrm{CONH}_{2}$ $\left(1700 \sim 1720 \mathrm{~cm}^{-1}\right)$ were reduced. The small shift in the asymmetric $\mathrm{NaSO}_{3}{ }^{-}$stretching vibration of from 1183 to 1188 $\mathrm{cm}^{-1}$ and $1045 \mathrm{~cm}^{-1}$ reinforces the hypothesis of dipole-ion interactions. This function machine appearance was dyed of reactive Blue 4 structure to make $\mathrm{NaSO}_{3}{ }^{-}$. From the changes of functional peaks shown in Fig. 1, the reactive blue 4 can be successfully immobilized on the specimens by the method we used in this experiment.

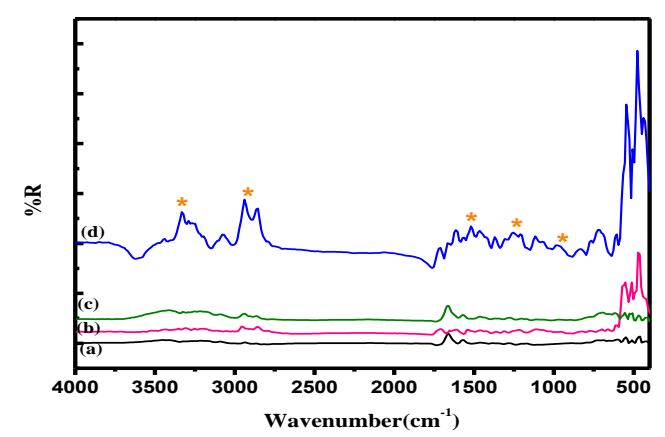

Fig. 1. FTIR spectra of (a) Control/dyeing 3 owf $\%$, treated (b) $\mathrm{O}_{2}$ Plamsa/dyeing 3 owf\%, treated (c) PD/graft-AAm 1wt \%/dyeing 3 owf\% treated.(d) PD/graft-AAm 10wt\%/dyeing 3 owf $\%$ treated.

\section{SEM Morphological Observation}

1) Nylon microfiber artificial leather surface morphology

Fig. 2 was the SEM micrographs of nylon microfiber artificial leather under the treatment below. Figure 2(a) showed the morphology of unmodified surface of nylon microfiber artificial leather was very smooth whiles a few microspores. Figure 2 (b) shows the unmodified dyeing after nylon microfiber artificial leather, the surface of nylon microfiber artificial leather are laminated shape and uniformity. By dyeing after oxygen plasma treatment observed the surface are covered by dye shown in Fig. 2 (c). Fig. 2 (d) shows the surface of dyeing after $\mathrm{O}_{2}$ plasma 
treatment and acryl amide grafting (10wt \%). We compared the morphology of after oxygen plasma induces post grafting co-polymerization acrylamide (AAm) on nylon microfiber artificial leather the surface were covered fully with fiber. This is an important finding that could lead to a new method for plasma graft polymerization.

\section{2) SEM micrograph versus dipping duration into water} for nylon microfiber artificial leather

Putting into nylon microfiber artificial leather water for 1min, after we observation surface state shown in Fig 2(e) and (f), The Fig 2(e) (d), shows the unmodified dyeing after nylon microfiber artificial leather via water durability, and dyeing after oxygen plasma treatment nylon microfiber artificial leather via water durability. In comparison, the surfaces are observes dye to turn condition. The Fig 2(f) with process of dyeing after $\mathrm{O}_{2}$ plasma treatment and acryl amide grafting (10wt \%) treatment is stable since the nice adhesion on surface with soaks in water the not obvious change. After Plasma graft polymerization process, the specimens undergoing oxygen plasma treatment AAm grafting, and dye immobilization had better absorbability and water durability.


Fig. 2. SEM micrographs of nylon microfiber artificial leather under the magnification of 1000x: (a) control (b) Control/dyeing 3 owf\%, treated (c) $\mathrm{O}_{2}$ Plamsa/dyeing 3 owf $\%$, treated (d) PD/graft-AAm 10wt\%/dyeing 3 owf $\%$ treated. (e) Control/dyeing 3 owf $\%$, water durability treated(f) $\mathrm{O}_{2}$

Plamsa/dyeing 3 owf\%, water durability treated (g) PD/graft-AAm10wt\% /dyeing 3 owf $\%$ water durability treated.

\section{Chemical Composition Analysis}

ESCA analysis was used to characterize the chemical bonding and the surface composition of the film. In this paper we will aim at dyeing after plasma graft polymerization. The surface elements of unmodified dyeing after nylon microfiber artificial leather were observed by ESCA measurements. As shown in Fig. 3(a), strong peaks of $\mathrm{C}, \mathrm{N}$, and $\mathrm{O}$ could be found on unmodified specimens. ESCA technique was applied to analyze the surface chemical composition of nylon microfiber artificial leather. The photoelectron peaks at $535.58 \mathrm{eV}, 402.7 \mathrm{eV}$ and $289.4 \mathrm{eV}$ correspond to the $\mathrm{O} 1 \mathrm{~s}, \mathrm{~N} 1 \mathrm{~s}$ and $\mathrm{C} 1 \mathrm{~s}$ orbits, respectively. Fig. 3(b) presents the ESCA survey spectra for the after dyeing control and the treated groups. Compared to the control, after dyeing have other photoelectron peaks at $\mathrm{Na} 1 \mathrm{~s}, \mathrm{Cl} 2 \mathrm{p}$, and $\mathrm{S} 2 \mathrm{p}$ orbits, respectively. Fig. 3(b) (c) is shown plasma graft polymerization process of dye on the nylon microfiber artificial leather. Many radicals could be found on the surface of the specimens after plasma modification. When the specimens were taken out from the chamber and connected with the atmosphere, peroxides would be formed on the surface; hence, the $O$ peak could be observed. Figure $3(d)$ shows the ESCA analyses of dyeing after $\mathrm{O}_{2}$ plasma treatment and acryl amide grafting (10wt $\%)$-modified specimens. The intensity (counts/s) of O1s can be obviously increased because the acryl amide was successful polymerized on the plasma-modified specimens. The element composition and the atomic ratios of $\mathrm{O} / \mathrm{C}$ and N/C derived from survey spectra for the four groups are given in Table I. The results of the element composition of control nylon microfiber artificial leather is in accordance with those describe in literature [2], [9], and [10]. The O/C ratios increased dramatically when the nylon microfiber artificial leather with $\mathrm{O}_{2}$ plasma treatment. When microfiber modified with plasma graft polymerization, the N/C ratios showed only small fluctuations for different groups after plasma modification.



Fig. 3. ESCA survey spectra (a) control (b) Control/dyeing 3 owf $\%$, treated (c) $\mathrm{O}_{2}$ Plamsa/dyeing 3 owf $\%$, treated (d) PD/graft-AAm 10wt $\% /$ dyeing 3 owf $\%$ treated.

TABLE I: ESCA ELEMENTAL ANALYSIS OF CONTROL AND WITH PLASMA GRAFT POLYMERIZATION NYLON MICROFIBER ARTIFICIAL LEATHER

\begin{tabular}{cccccccccc}
\multicolumn{3}{c}{ GRAFT POLYMERIZATION NYLON MICROFIBER ARTIFICIAL LEATHER } \\
\hline & \multicolumn{3}{c}{ Chemical composition (\%) } & \multicolumn{3}{c}{$\begin{array}{c}\text { Atomic } \\
\text { ratio (\%) }\end{array}$} \\
\cline { 2 - 7 } Sample & C1s & O1s & N1s & Na & Cl & S & O/C & N/C \\
\hline 1 & 80.25 & 16.25 & 3.5 & - & - & - & 20.2 & 4.36 \\
2 & 75.33 & 18.63 & 4.37 & 0.81 & 0.37 & 0.5 & 24.7 & 5.81 \\
3 & 73.79 & 22.84 & 1.67 & 0.7 & 0.15 & 0.85 & 30.9 & 7.3 \\
4 & 70.64 & 21.25 & 7.44 & 0.22 & 0.21 & 0.25 & 30.1 & 10.0 \\
\hline
\end{tabular}

\section{E. Dyeing Properties}

No matter under plasma deposition or use UV grafting polymerization, the grafting density increased with raises conc. After has dyed we can find graft quantity and dyeing density have the direct ratio. Graft quantity increases with the acryl amide concentration the measure of area dyeing density to reach $67.1\left(\mathrm{mg} / \mathrm{cm}^{2}\right)$, after water washed dye in reside 98.9\% were shown in Table II. The percent of dyeing density 
$=\left(W_{d}-W_{g}\right) / A^{2}$ (where $W_{g}$ is the weight of substrate before dyeing reactive Blue $4, \mathrm{Wg}$ is the weight of substrate after dyeing reactive Blue 4.) Fig.4 shows the shows $\mathrm{O}_{2}$ plasma treatment dyeing after Acryl amide grafting (10wt \%) treatment and water durability. The increased amount of amine end-groups induced by Plasma graft polymerization may play an important role in enhancing the formation of ionic linkage with dyes for nylon microfiber artificial leather, which speeded up the dye adsorption and reduced dye loss.

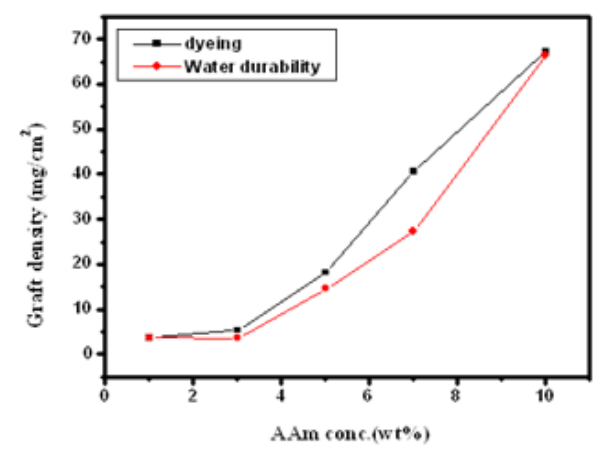

Fig. 4. Dry density of polymerization

TABLE II: DRY DENSITY AND WATER DURABILITY.

\begin{tabular}{|c|c|c|}
\hline \multirow{2}{*}{ Treatment } & \multicolumn{2}{|c|}{ Dyeing density $\left(\mathrm{mg} / \mathrm{cm}^{2}\right)$} \\
\cline { 2 - 3 } & Dyeing & Water reside $(\%)$ \\
\hline Original & 5.05 & 57.0 \\
\hline $\mathrm{O}_{2}$ plasma & 4.35 & 76.3 \\
\hline $\mathrm{PD} /$ graft-AAm $(1 \mathrm{wt} \%)$ & 3.72 & 97.5 \\
\hline $\mathrm{PD} /$ graft-AAm $(3 \mathrm{wt} \%)$ & 5.28 & 67.0 \\
\hline $\mathrm{PD} /$ graft-AAm $(5 \mathrm{wt} \%)$ & 18.1 & 80.1 \\
\hline $\mathrm{PD} /$ graft-AAm $(7 \mathrm{wt} \%)$ & 40.5 & 67.4 \\
\hline $\begin{array}{c}\text { PD /graft-AAm (10wt } \\
\%)\end{array}$ & 67.1 & 98.9 \\
\hline
\end{tabular}

\section{CONCLUSION}

Results from this study show that plasma treatment and UV-light induced grafting provided a good method for surface modification, which was suitable for materials of different appearances. In this study, the results of wettability indicate the porous nylon microfiber artificial leather following after UV-light induced grafting $\mathrm{O}_{2}$ plasma treatment will improve the hygroscopic of nylon microfiber artificial leather efficiency. The FTIR spectra and XPS of different modified nylon microfiber artificial leather showed that dyeing can be successfully immobilized on the specimens. Results from SEM morphology showed that modified nylon microfiber artificial leather had better to proceed dyeing. In conclusion, the Plasma graft polymerization process could contribute to promote absorbs of dye on the nylon microfiber artificial leather and increase colorfastness.

\section{REFERENCES}

[1] S. M. Burkinshaw and B. B. Allafan, "The development of a metal-free, tannic acid-based after treatment for nylon 6,6 dyed with acid dyes.Part 4: tannic acid," Dyes and Pigments, vol. 62, pp. 159-172, 2004

[2] L. Zhu, C. Wang, and Y. Qiu, "Influence of the amount of absorbed moisture in nylon fiberson atmospheric pressure plasma processing," Surface \& Coatings Technology, vol. 201, 2007, pp. 7453-7461

[3] H. Hocker, "Plasma treatment of textile fibers," Pure Appl. Chem., vol. 74, no. 3, pp. 423-427, 2002.

[4] J. H. Choi, E. S. Lee, H. K. Baik, S. J. Lee, K. M. Songc, M. K. Hwang, and C. S. Huh, "Surface modification of natural leather using low-pressure parallel plate plasma," Surface and Coatings Technology, vol. 171, 2003, pp. 257-263

[5] Y. A. Sona, J. P. Honga, H. T. Lima, T. K. Kimb, and D. Pigments, vol. 66, no. 3, pp. 231-239, September, 2005.

[6] K. S. Chen, "Surface Organic Modification of Inorganic Substrates by Plasma Deposition of Tin Oxide Organic-Like Thin Films and Grafting polymerization," Surface and Coatings Technology, vol. 200, 2006, pp. 3270-3277

[7] Q. Fan, S. Hoskote, and Y. Hou, "Reduction of colorants in nylon flock dyeing effluent," Journal of Hazardous Materials, vol.112, no. 1-2, pp. 123-131, 2004.

[8] H. S. Mukherjee and S. A. Jabrain, "Aging Characteristice of Oriented Poly(Ethylene Terephthalate)," Polymer Engineering and Science, vol.1, no. 35, no. 14, pp.1145-1154,1995.

[9] J. Tyczkowski, I. Krawczyk, and B. Woz niak, "Modification of styrene-butadiene rubber surfaces by plasma chlorination," Surface and Coatings Technology, pp. 174-175, 2003.

[10] G. Borcia, N. Dumitrascu, and G. Popa, Surf. Coat. Technol., vol. 197, no. $316,2005$.

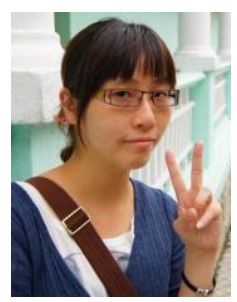

Shu-Chuan Liao received her PhD in 2011 under the supervi- sion of Professor Ko-Shao Chen at Tatung University. She was worked as a researcher fellow in the Center Thin Film Technologic and Applications, Ming Chi University of Technology, New Taipei City, Taiwan. Her research interests include the development of plasma polymerization, Surface Modification, Atmospheric Plasma, Smart Hydrogel, biomedical application, and Gas/Bio/Humidity sensor, Plasma technique (PECVD) for engineering applications, Micro-Arc Oxidation (MAO), ICP-CVD.

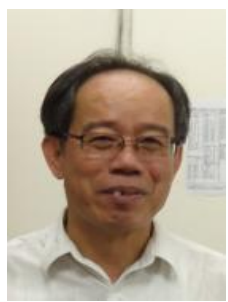

Ko-Shao Chen received his $\mathrm{PhD}$ in 1983 at the Shizuoka University of electronic materials science. $\mathrm{He}$ worked as an associate professor in the department of Materials Engineering, Tatung Institute of Technology Taiwan. He joined visited scholar at Kyoto University of the Research Center for Biomedical Engineering. He is a professor in the department of Materials Engineering, Tatung University, Taipei, Taiwan.

His research interests include the development of the Surface Modification (plasma treated and UV grafted polymerization), Atmospheric Plasma, Smart Hydrogel, biomedical application, and Gas/Bio/Humidity sensor, Plasma technique (PECVD) for engineering applications.

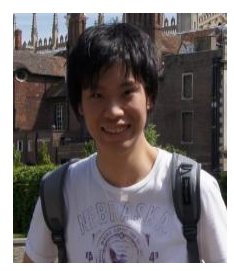

Wei-Yu Chen received his master in 2011 at Institute of Biomedical Engineering, National Taiwan University, Taipei, Taiwan He is involved in several research projects including the design and synthesis of biomaterial.

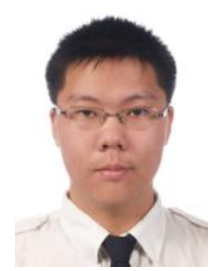

Chin-Yen Chou is a undergraduate student from the Department of Materials Engineering since January 2010 under the guidance of Professor Ko-Shao Chen at Tatung University. He is involved in several research projects including the design and synthesis of thermal sensitive layer, silicon oxide barrier films for sensing device applications.

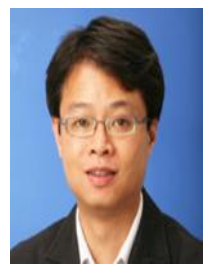

Chi Wai Kan graduates from Hong Kong Polytechnic University with a BSc and $\mathrm{PhD}$ in Textiles Chemistry. Dr. Kan is now an Associate Professor in institute of Textiles and Clothing, The Hong Kong Polytechnic University. His research interests include the surface modification of textile material with the use of novel technology such as plasma and laser. 